\title{
Arcobacter butzleri
}

National Cancer Institute

\section{Source}

National Cancer Institute. Arcobacter butzleri. NCI Thesaurus. Code C86153.

A species of Gram negative, rod shaped bacteria assigned to the phylum Proteobacteria.

This bacteria is oxidase positive, catalase variable, motile by one or more polar flag ella,

non spore forming and reduces nitrate. A. butzleri is found in untreated water and slaughter houses and is an emerging foodborne pathogen. 